\title{
Avaliação do estado nutricional e do desenvolvimento neuropsicomotor em crianças freqüentadoras de creche
}

\author{
Nutritional status and neurodevelopment of children enrolled in a day care center
}

Terezinha Soares Biscegli', Larissa Bueno Polis², Lívia Marcela dos Santos², Mariana Vicentin ${ }^{2}$

\section{RESUMO}

Objetivo: Avaliar o estado nutricional e o desenvolvimento neuropsicomotor em crianças freqüentadoras de uma creche.

Métodos: Estudo transversal de 113 crianças com seis a 70 meses de idade, que freqüentavam a creche Irmã Sheila em Catanduva, São Paulo. A avaliação nutricional foi feita por antropometria e analisada pela classificação de Waterlow e a avaliação do desenvolvimento neuropsicomotor pelo Teste de Denver II, em todas as crianças incluídas no estudo. Dados adicionais foram obtidos por meio de um questionário respondido pelos pais em $70 \%$ dos casos.

Resultados: Entre as crianças estudadas, observou-se $12 \%$ de desnutrição aguda, $1 \%$ de desnutrição pregressa e $16 \%$ de obesidade. O Teste de Denver II detectou 37\% de suspeitos de atraso no desenvolvimento, sendo a linguagem a área mais acometida. A faixa etária mais comprometida foi a de 25 a 60 meses. O questionário mostrou que $87 \%$ das crianças receberam aleitamento materno ( $57 \%$ até o sexto mês), $96 \%$ das mães trabalhavam fora de casa, $54 \%$ delas possuíam ensino médio completo e $73 \%$ das famílias tinham renda máxima de dois salários-mínimos.

Conclusões: A alta prevalência de distúrbios nutricionais e possíveis atrasos no desenvolvimento observados em crianças da creche Irmã Sheila mostraram a necessidade de introduzir dietas balanceadas, incentivando o desenvolvimento de hábitos alimentares saudáveis, além de alertar para a questão da interferência negativa dos fatores socioeconômicos e culturais no crescimento e desenvolvimento infantil.

Palavras-chave: criança; creches; estado nutricional; desenvolvimento infantil.

\section{ABSTRACT}

Objective: Evaluate the nutritional status and neurodevelopment of children enrolled in a day care center.

Methods: This cross-sectional study enrolled 113 children aged six to 70 months, assisted in a nonprofit day care center in Catanduva, São Paulo. All children were submitted to evaluation of the nutritional status by anthropometry and classified according to Waterlow criteria. Child development was assessed according to Denver II Test. Further characteristics were obtained by a questionnaire answered by $70 \%$ of the children's parents.

Results: Among the evaluated children, $12 \%$ had acute malnutrition, $1 \%$ past malnutrition and $16 \%$ obesity. The Denver II Test detected $37 \%$ of children with suspect of development delay (the language was the ability area more frequently affected). Children aged 25 to 60 months had more delays. Data from the questionnaire showed that $87 \%$ of children received breastfeeding ( $57 \%$ until around the sixth month of age), $96 \%$ of the mothers worked outside home, $54 \%$ of them had high school and $73 \%$ of the families received less than two minimum wages.

Conclusions: The high prevalence of nutritional problems and possible developmental delays observed in this study indicates the need of adopting balanced diets and improving education towards healthier alimentary habits. Attention should be given in order to act on the negative influence of the poor socioeconomic and educational background on children's growth and development.

Key-words: child; child day care centers; nutritional status; child development.

Endereço para correspondência:

Terezinha Soares Biscegli

Rua Fortaleza, 95 - Jardim Ferreira

CEP 15840-000 - Itajobi/SP

E-mail: terezinhabiscegli@yahoo.com.br

Recebido em: 25/7/2007

Aprovado em: 18/9/2007 


\section{Introdução}

Segundo a Lei de Diretrizes e Bases da Educação Nacional (LDBEN, lei 9.394/96), creches são instituições que atendem a crianças de zero a três anos e pré-escolares de quatro a seis anos. As duas faixas etárias compreendem a educação infantil, que é a primeira etapa da educação básica e tem como finalidade o desenvolvimento integral da criança em seus aspectos físico, psicológico, intelectual e social ${ }^{(1)}$.

Sabe-se que os primeiros anos de vida - especialmente os três iniciais - são cruciais para a aquisição de conhecimentos e habilidades, motivo pelo qual é importante promover o desenvolvimento infantil nessas instituições, onde as crianças permanecem por longos períodos e durante uma fase fundamental do seu crescimento e desenvolvimento ${ }^{(2-5)}$.

A creche vem se tornando uma necessidade significativa da população, em conseqüência das transformações socioeconômicas que a sociedade está sofrendo. Como as crianças permanecem na creche de oito a dez horas por dia e, durante este tempo, recebem dois terços de suas necessidades nutricionais, além da orientação psicopedagógica, é necessário que a alimentação e os cuidados oferecidos satisfaçam suas necessidades e influenciem favoravelmente o seu estado nutricional e desenvolvimento neuropsicomotor ${ }^{(6)}$.

O estado nutricional é conseqüência de vários fatores, de modo que é importante a influência exercida pelo meio ambiente. A vigilância nutricional constante faz-se necessária nos países em desenvolvimento, devido à alta prevalência de distúrbios como a desnutrição e, mais recentemente, a obesidade ${ }^{(7)}$. Portanto, a avaliação do estado nutricional é etapa fundamental no estudo de uma criança, para verificar se o crescimento está se afastando do padrão esperado devido a alguma doença e/ou condições sociais desfavoráveis. Nutricionalmente, o período entre o desmame e os cinco anos de idade é a fase mais vulnerável da vida de uma criança ${ }^{(8)}$.

Vários são os fatores relacionados à gênese da desnutrição. Destacam-se, dentre eles, a desnutrição intra-uterina, a desnutrição pós-natal, a prematuridade, o abandono precoce do aleitamento materno, as infecções repetidas, além do fraco vínculo mãe-filho ${ }^{(9)}$. A obesidade infantil, cuja incidência vem aumentando em nosso meio, com grave prognóstico, também é um fator a ser considerado ${ }^{(10)}$.

Outro aspecto a ser avaliado é o desenvolvimento neuropsicomotor. Cerca de uma em cada oito crianças apresenta alterações do desenvolvimento que podem interferir de forma significativa em sua qualidade de vida e inclusão social. O desenvolvimento é o resultado da interação entre as características biológicas da criança e os fatores culturais e sociais em que ela está inserida. Assim, a aquisição de novas habilidades relaciona-se à faixa etária e às interações vividas com os outros indivíduos do seu grupo. A avaliação do desenvolvimento é, portanto, um processo individualizado, dinâmico e compartilhado com cada criança.

Um dos instrumentos para essa avaliação é o Teste de Triagem do Desenvolvimento de Denver (TTDD) ou simplesmente Teste de Denver ${ }^{(11)}$. É utilizado pelos profissionais da área da Saúde para triagem em populações assintomáticas, pois permite fácil treinamento e rápida aplicação ${ }^{(12)}$. Consiste em 125 itens distribuídos em quatro áreas do desenvolvimento: pessoal-social (aspectos da socialização da criança dentro e fora do ambiente familiar), motor fino (coordenação olho/mão, manipulação de pequenos objetos), linguagem (produção de som, capacidade de reconhecer, entender e usar a linguagem) e motor grosseiro (controle motor corporal como sentar e andar).

Os dados obtidos no teste são utilizados para determinar se a criança está progredindo como esperado para sua idade cronológica e maturidade, assim como para planejar estratégias de atuação junto à criança, podendo sugerir a necessidade de um exame pormenorizado, com conseqüente encaminhamento a profissionais especializados ${ }^{(13,14)}$.

Em decorrência dos fatos expostos, o objetivo do presente estudo foi avaliar o estado nutricional e o desenvolvimento neuropsicomotor das crianças freqüentadoras da creche Irmã Sheila, localizada na cidade de Catanduva, em São Paulo.

\section{Métodos}

Estudo de corte transversal realizado em junho de 2007. Foram incluídas as crianças admitidas na creche Irmã Sheila, sendo classificadas como de "baixa renda" de acordo com os critérios estabelecidos pela própria instituição, a qual leva em consideração a renda familiar, o tipo de moradia, a composição familiar e, principalmente, a necessidade da mãe trabalhar fora de casa.

O presente projeto de pesquisa foi aprovado pelo Comitê de Ética da Faculdade de Medicina de Catanduva e foi solicitada a assinatura do termo de consentimento livre e esclarecido pelos responsáveis legais das crianças. 
Das 120 crianças matriculadas na creche, uma foi excluída do estudo por apresentar doença capaz de interferir no processo de crescimento e desenvolvimento (cardiopatia congênita), três por não comparecerem nos dias de avaliação e três por não terem o consentimento dos pais para participar do estudo.

Determinou-se o estado nutricional e aplicou-se o Teste de Denver nas 113 crianças de seis a 70 meses incluídas no estudo. Um questionário com dados adicionais sobre a criança foi entregue aos responsáveis para ser preenchido, obtendo-se resposta em $70 \%$ dos casos.

A ficha de matrícula da criança foi utilizada para determinar a idade cronológica (em meses) e o sexo. A antropometria (peso e estatura) obedeceu às técnicas preconizadas pela Organização Mundial da Saúde ${ }^{(15,16)}$ e foi realizada exclusivamente por um dos três autores especialmente treinado para desempenhar tal tarefa. A medição do comprimento da criança de zero a 24 meses foi feita com a criança em decúbito dorsal (antropômetro horizontal) e, para as maiores de dois anos, aferiu-se a altura em posição ortostática (antropômetro vertical). A aferição do peso foi realizada com balança pediátrica (precisão de $10 \mathrm{~g}$ ) e balança plataforma (precisão de $100 \mathrm{~g}$ ) para crianças de até $15 \mathrm{~kg}$ ou acima de $15 \mathrm{~kg}$, respectivamente.

Para a avaliação nutricional, utilizou-se a classificação de Waterlow $^{(17)}$, que leva em conta as relações entre peso/altura (P/A) e altura/idade (A/I), sendo os pontos de corte de $80 \mathrm{e}$ 90\% da média, respectivamente, para definir desnutrição. Foram consideradas eutróficas as crianças que apresentaram $\mathrm{P} / \mathrm{A}$ e A/I normais; com desnutrição aguda, as que apresentaram P/A abaixo do ponto de corte e A/I normal; e com desnutrição pregressa as que apresentaram P/A normal e A/I abaixo do ponto de corte. Foram classificadas como obesas as crianças que apresentaram P/A acima de $120 \%$, calculando-se o índice de massa corpórea (IMC) de cada uma. A obesidade foi confirmada quando o IMC foi igual ou superior ao percentil $95^{(18)}$. As avaliações foram feitas para cada criança, individualmente e de forma manual, utilizando-se as tabelas de referência do National Center for Health Statistics, 2000 ${ }^{(19)}$.

A avaliação do desenvolvimento neuropsicomotor foi realizada pelo Teste de Denver II ${ }^{(11)}$, aplicado individualmente para cada criança por um dos três pesquisadores especialmente treinados. O teste foi aplicado na própria creche, em sala previamente preparada. Na aplicação do Teste de Denver II, foram considerados alguns fatores de exclusão temporária que poderiam afetar o desempenho do paciente (sono, fadiga, adoecimento, febre ou medo). Durante a avaliação, o teste era interrompido caso a criança se cansasse ou precisasse participar de alguma atividade prevista na creche ${ }^{(20)}$. Após a análise de cada item ou comportamento, o teste global teve os seguintes resultados: normal, quando não havia atrasos ou no máximo uma cautela; suspeita de atraso, quando havia duas ou mais cautelas e/ou um ou mais atrasos.

\section{Resultados}

Das 113 crianças estudadas, 43 (38\%) eram do sexo masculino. A idade variou de seis a 70 meses. A faixa etária predominante foi a de 25 a 60 meses, com 85 crianças ( $75 \%$ dos casos).

De acordo com as respostas aos questionários e considerando-se algumas abstenções em determinados itens, 60 $(87 \%)$ crianças receberam aleitamento materno $(57 \%$ até o sexto mês). Em 71 (96\%) domicílios, a mãe trabalhava fora de casa; dessas, 41 (54\%) possuíam ensino médio completo e 57 (73\%) famílias tinham renda máxima de dois salários-mínimos.

A avaliação do estado nutricional está demonstrada na Tabela 1. A desnutrição predominou na faixa etária de 25 a 60 meses (oito crianças, 57\%), seguida da faixa de zero a 24 meses (cinco crianças, 36\%), e, finalmente, entre as crianças menores de um ano ou entre aquelas com 61 meses ou mais, apenas uma (7\%) em cada grupo apresentou desnutrição. Não houve diferença de prevalência de desnutrição entre os sexos e não foi encontrado nenhum caso de desnutrição crônica. A obesidade foi mais freqüente no sexo feminino

Tabela 1 - Distribuição das crianças de acordo com o estado nutricional

\begin{tabular}{lcc}
\hline Estado nutricional & $\begin{array}{c}\text { Número de } \\
\text { crianças }\end{array}$ & Porcentagem \\
\hline Eutrofia & 81 & $71,7 \%$ \\
Desnutrição aguda & 13 & $11,5 \%$ \\
Desnutrição pregressa & 1 & $0,9 \%$ \\
Obesidade & 18 & $15,9 \%$ \\
\hline
\end{tabular}

Tabela 2 - Distribuição das crianças de acordo com o resultado do Teste de Denver II, segundo o estado nutricional

\begin{tabular}{lcc}
\hline Estado nutricional & $\begin{array}{c}\text { Denver II } \\
\text { suspeito (n) }\end{array}$ & Porcentagem \\
\hline Eutrofia & 30 & $37 \%$ \\
Desnutrição aguda & 5 & $38 \%$ \\
Desnutrição pregressa & 1 & $100 \%$ \\
Obesidade & 6 & $33 \%$ \\
\hline
\end{tabular}


$(61 \%)$ e foi observada em 17 (94\%) crianças na faixa de 25 a 60 meses e em uma (6\%) com mais de 60 meses.

Analisando-se os resultados globais do Teste de Denver II, observou-se que 42 das 113 crianças estudadas (37\%) apresentaram resultado suspeito. Destas, 25 (22\%) apresentaram alteração em apenas uma área do desenvolvimento, oito (7\%) em duas áreas, três (3\%) em três e seis (5\%) em todas elas.

A distribuição das crianças de acordo com os resultados do Teste de Denver II, segundo áreas do desenvolvimento, está demonstrada no Gráfico 1: a área mais comprometida foi a da linguagem. Com relação à faixa etária, 29 (25\%) casos suspeitos foram encontrados em crianças de 25 a 60 meses, 11 (10\%) em menores de dois anos, e dois (2\%) em maiores de cinco anos. $\mathrm{Na}$ Tabela 2, observa-se a prevalência de resultado suspeito no Teste de Denver II, de acordo com o estado nutricional.

\section{Discussão}

A análise dos dados antropométricos das crianças freqüentadoras da creche Irmã Sheila revelou que houve maior prevalência de obesidade do que desnutrição, seja esta aguda ou pregressa. Resultados semelhantes foram observados em outros estudos que evidenciaram um aumento da obesidade em todas as camadas da população, mas, principalmente, em famílias de menor poder aquisitivo $^{(21)}$. Este fato já é motivo de preocupação em nível de Saúde Pública, pois, a presença de obesidade leva a um aumento das taxas de morbidade e de doenças crônicas como diabetes, doenças cardiovasculares, problemas ortopédicos e distúrbios psicológicos e sociais $^{(6)}$. Neste sentido, nos últimos 30 anos, houve mudança nos padrões nutricionais da população brasileira (transição nutricional), com evidente diminuição de desnutridos e aumento da população de indivíduos com sobrepeso ou obesidade, principalmente devido aos hábitos alimentares inadequados ${ }^{(22)}$.

Embora venha ocorrendo redução da prevalência de desnutrição calórico-protéica em crianças menores de cinco anos nas últimas décadas, no Brasil esta é ainda considerada um problema de Saúde Pública, principalmente nas regiões Nordeste e Norte, nos denominados bolsões de pobreza ${ }^{(23)}$. Em nossa pesquisa, encontramos $12 \%$ de crianças com desnutrição aguda, $1 \%$ com desnutrição pregressa e $16 \%$ com obesidade. A baixa prevalência de desnutrição nas crianças menores de dois anos, sobretudo nos abaixo de um ano, pode se relacionar aos altos índices de aleitamento materno praticado durante os primeiros anos de vida (87\%). A desnutrição aguda reflete uma deficiência nutricional recente, com perda significativa de peso corpóreo, podendo estar associada a várias doenças. Por outro lado, a desnutrição crônica caracteriza-se por baixa estatura para a idade, refletindo deficiências cumulativas de saúde e nutrição durante um longo período ${ }^{(1)}$.

Resultados diferentes foram encontrados em crianças de creches beneficentes do município de São Paulo (1\% de desnutrição atual, $6 \%$ de desnutrição crônica e $6 \%$ de obesidade $)^{(24)}$. Entretanto, dados similares aos do presente estudo foram observados por Corso $e t a l^{(25)}$, que detectaram

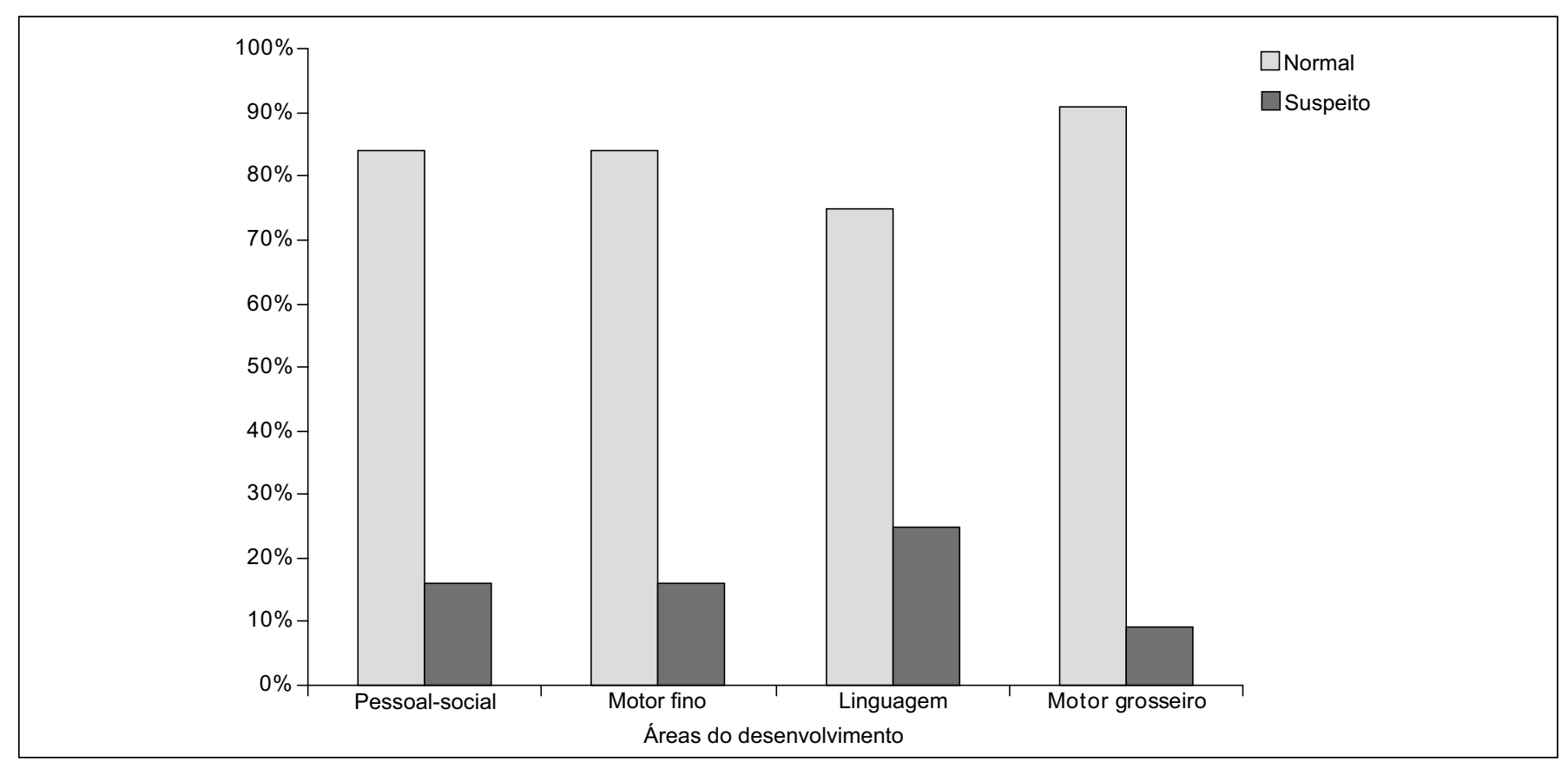

Gráfico 1 - Distribuição das crianças de acordo com os resultados do Teste de Denver II, segundo as áreas do desenvolvimento 
$2 \%$ de desnutrição aguda e $7 \%$ de sobrepeso/obesidade, em crianças menores de seis anos, na cidade de Florianópolis, em Santa Catarina. Em pesquisa desenvolvida com crianças de ambulatório pediátrico, Torres et al ${ }^{(26)}$ detectaram, em maiores de dois anos, 9\% de desnutrição aguda, 15\% de desnutrição pregressa, $3 \%$ de desnutrição crônica e $15 \%$ de sobrepeso/obesidade. Tal divergência de resultados possivelmente se explica pelo fato de se tratarem de populações diversas, avaliadas por critérios diferentes.

Estudo realizado em uma creche beneficente de Catanduva mostrou que $4 \%$ das crianças apresentavam desnutrição aguda, $2 \%$, desnutrição crônica e $7 \%$, obesidade ${ }^{(6)}$. Embora a creche do estudo referido e a aqui analisada sejam beneficentes e estejam situadas na mesma cidade, a diferença notada pode ser devida ao fato de a última se localizar em bairro de periferia, abrigando crianças de situação socioeconômica menos favorecida.

Crianças freqüentadoras de creche recebem praticamente $70 \%$ de suas necessidades nutricionais durante o período de permanência nessas instituições e, por receberem uma alimentação balanceada durante praticamente dez horas do dia, deveriam estar mais protegidas da desnutrição, quando comparadas às crianças da população em geral ${ }^{(27,28)}$. Daí a importância do fornecimento de uma dieta equilibrada, com quantidades suficientes de nutrientes para prevenir alterações nutricionais. A alimentação oferecida às crianças deste estudo foi preparada pela cozinha da prefeitura municipal, que obedece a critérios estabelecidos por um nutricionista. Trata-se de dieta geral, adequada às diferentes faixas etárias.

De acordo com os resultados observados em nosso estudo, verificamos que tanto a prevalência de desnutrição como a de obesidade está concordante com a média populacional, mas chama a atenção que a freqüência de obesidade está aumentando na população de baixa renda.

A análise dos resultados globais obtidos pela aplicação do Teste de Denver II mostrou que parte significativa da população estudada apresentou resultado suspeito (37\%). Cabe ressaltar que foram encontrados poucos estudos brasileiros de triagem do desenvolvimento de crianças usando o Teste de Denver II $^{(29-33)}$. Tais estudos são, em geral, transversais, em diferentes faixas etárias e realizados em locais diversos (creches públicas e privadas, domicílio e ambulatórios), dificultando a comparação dos resultados.

Beteli ${ }^{(3)}$ e Rezende $e t$ a ${ }^{(34)}$ utilizaram a mesma metodologia em seus estudos e observaram resultados globais idênticos (em torno de $20 \%$ de suspeito). No trabalho de Beteli(3), as crianças de zero a seis anos pertenciam a uma população economicamente privilegiada e, no de Rezende et al ${ }^{(34)}$, os pacientes de zero a dois anos eram de classe baixa. Resultados semelhantes também foram encontrados em outra pesquisa com crianças de zero a dois anos que freqüentavam creches públicas de Florianópolis ${ }^{(29)}$. Pesquisadores de Pelotas, no Rio Grande do Sul, estudando crianças de um ano de idade, observaram prevalência de 34\% de casos suspeitos no resultado global do Teste de Denver II. As crianças mais pobres apresentaram o dobro de falhas no Teste de Denver II, comparadas às de renda maior ${ }^{(32)}$.

Com relação ao sexo das crianças, não houve diferença significante quanto ao resultado global do teste (37\% suspeitos no feminino e $40 \%$ no masculino). Resultados similares foram verificados em outros estudos ${ }^{(30,31)}$.

Analisando-se concomitantemente os resultados globais do Teste de Denver II e o estado nutricional, não houve diferenças significantes na prevalência de casos suspeitos entre a população eutrófica, com desnutrição aguda e com obesidade. Um estudo com crianças de zero a 12 meses de idade do Rio Grande do Norte revelou que o desempenho no Teste de Denver II não foi afetado pela condição nutricional ${ }^{(30)}$.

Ao avaliar separadamente as diversas áreas do desenvolvimento, a mais comprometida no presente estudo foi a linguagem, seguida por pessoal-social, motor fino-adaptativo e motor grosseiro. Resultados semelhantes foram observados por outros autores ${ }^{(29,33,34)}$. Um estudo realizado na Região Metropolitana de São Paulo demonstrou que, quanto mais elevado é o nível de escolaridade das mães, maior é o seu conhecimento acerca do desenvolvimento da linguagem infantil ${ }^{(35)}$. Em nossa pesquisa, a quase totalidade das mães trabalhava fora de casa e, no que se refere à escolaridade, apenas metade delas possuía ensino médio completo.

A alta prevalência de casos com resultado suspeito de atraso no desenvolvimento neuropsicomotor observado nessa pesquisa alerta para um risco potencial de atraso, o qual deve ser confirmado por testes diagnósticos. Assim, as crianças com resultado suspeito foram encaminhadas a profissionais especializados para avaliação mais acurada e intervenções pertinentes.

Pode-se concluir que a prevalência elevada de desnutrição e obesidade mostra a necessidade de medidas preventivas, como a implementação de dietas mais balanceadas e a prática de orientação nutricional, ensinando e estimulando hábitos alimentares saudáveis e, conseqüentemente, reduzindo a prevalência de distúrbios como obesidade e desnutrição. Por outro lado, a alta prevalência de casos suspeitos de alterações no desenvolvimento alerta para a questão da qualidade no cuidado com a criança e a interferência negativa dos fatores 
socioeconômicos e culturais no crescimento e desenvolvimento infantil, tanto no que se refere à instituição creche como ao âmbito familiar. Possivelmente, uma maior interação dos profissionais da creche com os pais das crianças, por meio de reuniões, palestras e atividades sociais, permitiria maior compreensão das necessidades e deficiências de cada criança, possibilitando personalizar estratégias de intervenção. Investir em capacitação pessoal e material pedagógico também poderia contribuir para minimizar os distúrbios observados. Dessa forma, a creche deixaria de ser uma instituição de caráter assistencial para ser uma promotora de saúde infantil.

\section{Agradecimentos}

À senhora Maria Antônia Barca Paschoa, coordenadora da creche Irmã Sheila, pela receptividade e auxílio na parte operacional, e aos professores, funcionários, pais e crianças, pela participação e interesse.

\section{Referências bibliográficas}

1. Brasil - Ministério da Educação [homepage on the internet]. Diretrizes e bases da educação nacional. Lei nº. 9.394, 1996. [cited 2007 Jul 13]. Available from: http://www.planalto.gov.br/ccivil_03/Leis/L9394.htm

2. Shore R. Repensando o cérebro: novas visões sobre o desenvolvimento inicial do cérebro. Porto Alegre: Mercado Aberto; 2000.

3. Beteli VC. Acompanhamento do desenvolvimento infantil em creches [tese de mestrado]. São Paulo (SP): USP; 2006.

4. Campbell FA, Ramey CT. Effects of early intervention on intellectual and academic achievement: a follow up study of children from low-income families. Child Dev 1994;65: 684-98.

5. Ferreira AMA. Prevenção da anemia ferropriva em lactentes que freqüentam creches no município de São Paulo, através de uma fórmula láctea fortificada com ferro [tese de doutorado]. São Paulo (SP): Unifesp; 2000.

6. Biscegli TS, Corrêa CE, Romera J, Hernandez JL. Avaliação do estado nutricional e prevalência de carência de ferro em crianças freqüentadoras de uma creche. Rev Paul Pediatr 2006;24:323-9.

7. Unicef/UNU/WHO/MI [Homepage on the internet]. Preventing iron deficiency in women and children: background and consensus on key technical issues and resources for advocacy, planning and implementing national programs. New York: International Nutrition Foundation and Micronutrient Initiative 1998. p. 1-60 [cited $2007 \mathrm{Jul}$ 13]. Available from: http://www.inffoundation. org/pdf/prevent_iron_def.pdf

8. Monte C. Desnutrição: um desafio secular à saúde infantil. J Pediatr (Rio J) 2000;76(Suppl 3):S285-97.

9. Nóbrega FJ, Campos ALR, Nascimento CFL. Vínculo mãe/filho na desnutrição. In: Nóbrega FJ, Campos ALR, Nascimento CFL, editores. Distúrbios nutricionais e fraco vínculo mãe/filho. $2^{\text {a }}$ ed. Rio de Janeiro: Revinter; 2000. p. 55-65.

10. Comitê de Nutrição da Sociedade de Pediatria de São Paulo. Avaliação do estado nutricional das crianças. Rev Paul Pediatr 1992;10:102-3.

11. Frankenburg KW, Dodds J, Archer P, Bresnick B, Maschka P, Edelman $\mathrm{N}$ et al. Denver II: Technical manual and training manual. Denver: Denver Developmental Materials; 1990.

12. Klausing KR, Duca MF, Neves MC. Dificuldades encontradas na aplicação do teste de triagem do desenvolvimento de Denver pelos alunos do projeto Creche das Rosinhas. Anais do $7^{\circ}$ Encontro de Extensão da Universidade Federal de Minas Gerais; 2004 Set 12-15; Belo Horizonte, MG. p.1-8.

13. Sociedade Brasileira de Pediatria [homepage on the Internet]. Desenvolvimento infantil [cited 2007 Jul 13]. Available from: http://www.sbp.com. br/show_item2.cfm?id_categoria=17\&id_detalhe=1967\&tip

14. Brêtas JRS. Avaliação psicomotora de crianças de 5 a 7 anos de idade, que freqüentam a creche "Maria Aparecida Carlini", Jardim Sabiá, Município de São Paulo [dissertação de mestrado]. São Paulo (SP): Unifesp; 1991

15. World Health Organization. Physical status: the use and interpretation of anthropometry. World Health Org Tech Rep 1995;854:1-452.

16. Hamill PV, Drizd TA, Johnson CL, Reed RB, Roche AF. NCHS growth curves for children birth-18 years. Vital Health Stat 1977;11:1-74.

17. Waterlow JC, Buzina R, Keller W, Lane JM, Nichaman MZ, Tanner JM. The presentation and use of height and weight data for comparing the nutritional status of groups of children under the age of 10 years. Bull World Health Organ 1977;55:489-98.

18. Rosner B, Prineas R, Loggie J, Daniels SR. Percentiles for body mass index in U.S. children 5 to 17 years of age. J Pediatr 1998;132:221-2.

19. NCHS [homepage on the Internet]. 2000 CDC growth charts: United States [cited 2007 Sep 7]. Available from: http://www.cdc.gov/growthcharts/

20. Rezende MA, Lima FG, Beteli VC, Santos JLF. Habilidades de linguagem e pessoal social de crianças de 0 a 3 anos de idade cuidadas em creches. Rev Bras Cresc Desenv Hum 2003;13:40-53.

21. Taddei JA, Colugnati FA, Rodrigues EM. Transição nutricional em menores de 5 anos: evidência dos inquéritos antropométricos brasileiros. In: Cardoso AL, Lopes LA, Taddei JA, editores. Tópicos atuais em nutrição pediátrica. São Paulo: Atheneu; 2004. p. 11-43.

22. Batista Filho $M$, Rissin $A$. A transição nutricional no Brasil: tendências regionais e temporais. Cad Saude Publica 2003;19(Suppl 1):S181-91.

23. Monteiro CA, Conde WL. Tendência secular da desnutrição e da obesidade na infância na cidade de São Paulo (1974-1996). Rev Saude Publica 2000;34:52-61.

24. Machado EH, Brasil AL, Palma D, Taddei JA. Condição nutricional e prevalência de anemia em crianças matriculadas em creches beneficentes. Rev Paul Pediatr 2005;23:21-6.

25. Corso AC, Botelho JL, Zeni LA, Moreira EA. Sobrepeso em crianças menores de seis anos de idade em Florianópolis, SC. Rev Nutr 2003;16:21-8.

26. Torres MA, Queiroz SS, Schvartsman C, Santos E, Nóbrega FJ. Prevalência de anemia e avaliação da condição nutricional de crianças atendidas no Ambulatório de Pediatria do Programa Einstein na comunidade de Paraisópolis. Rev Paul Pediatria 2002;20:61-8.

27. Silva LS, Giuglian ER, Aerts DR. Prevalência e determinantes de anemia em crianças de Porto Alegre, RS, Brasil. Rev Saude Publica 2001;35:66-73.

28. Taddei JA, Cannon MJ, Warner L, Souza P, Vitalle S, Palma D et al. Nutritional gains of underprivileged children attending a day care center in Sao Paulo City, Brazil: a nine month follow-up study. Rev Bras Epidemiol 2000;3:29-37.

29. Caon G, Ries LGK. Suspeita de atraso no desenvolvimento neuropsicomotor em idade precoce: uma abordagem em creches públicas. Temas Desenvolv 2003;12:11-7.

30. Cunha HL. Desenvolvimento de crianças atendidas no hospital de pediatria da Universidade Federal de Rio Grande do Norte no primeiro ano de vida: aplicação do Teste de Denver Il em ambulatório [tese de mestrado]. São Paulo (SP): Unifesp; 2000.

31. Halpern R, Barros FC, Horta BL, Victora CG. Desenvolvimento neuropsicomotor aos 12 meses de idade em uma coorte de base populacional no Sul do Brasil: diferenciais conforme peso ao nascer e renda familiar. Cad Saude Publica 1996;12:73-8.

32. Halpern R, Giugliani ER, Victora CG, Barros FC, Horta BL. Fatores de risco para suspeita de atraso no desenvolvimento neuropsicomotor aos 12 meses de vida. J Pediatr (Rio J) 2000;76:421-8.

33. Souza ABG, Siqueira CN. Avaliação do desenvolvimento de um grupo de crianças assistidas em creche, usando o teste de triagem de Denver II. Enferm Brasil 2003;2:96-103.

34. Rezende MA, Beteli VC, Santos JLF. Avaliação de habilidades de linguagem e pessoal-sociais pelo Teste de Denver II em instituições de educação infantil. Acta Paul Enferm 2005;18:56-63.

35. Cardoso RM. Conhecimento de mães e auxiliares de desenvolvimento infantil referente ao desenvolvimento de linguagem de crianças de 0 a 24 meses de idade [tese de mestrado]. São Paulo (SP): Unifesp; 2001. 